\title{
Arbeitsort Privathaushalt: eine arbeitsschutzfreie Zone?
}

\author{
MARTA BÖNING
}

Seit Jahrhunderten verdienen Menschen durch Arbeit in Haushalten anderer ihr Brot. ${ }^{1}$ Die Rechtsentwicklung schreitet voran, eins hat sich dennoch nicht geändert: Hausangestellte arbeiten in einem Umfeld, welches sich den Mechanismen externer Kontrolle weitgehend entzieht. Was im Haushalt passiert, bleibt in der Regel im Haushalt. Die Wahrscheinlichkeit, dass Hausangestellte die Verletzung ihrer Rechte belegen können, ist sehr gering. Hausangestellte klagen deshalb nicht. Und auch der Staat und die Versicherungsträger mit ihren Kontrollen greifen nur ausnahmsweise in diesen durch Art. 13 GG grundrechtlich geschützten Raum ein. Beschäftigte in Haushalten, so die Schätzungen von Experten, arbeiten zu 90 \% irregulär. Kommt es zur Verletzung von geltenden Schutzvorschriften, bekommt es kaum jemand mit. Das gilt erst recht, wenn Arbeits- und Wohnort zusammenfallen.

Hausangestellte, die in den Haushalten ihrer Arbeitgeber wohnen, zu Neudeutsch als „Live-ins“ bezeichnet, waren infolge der Demokratisierungsprozesse nach dem Zweiten Weltkrieg aus den deutschen Haushalten so gut wie verschwunden. Seit einiger Zeit sind „Live-ins“ wieder im Kommen, vorangetrieben durch die Alterung der deutschen Gesellschaft auf der einen und die berufliche Emanzipation der Frauen auf der anderen Seite. Immer mehr Pflegebedürftige können nicht (mehr) damit rechnen, dass Angehörige die Hauptlast der häuslichen Pflege auf sich nehmen. Lösungen seitens des staatlichen Pflegesystems für die Versorgung älterer Menschen in ihren Haushalten sind unzureichend. Gleichzeitig treibt die schwierige Arbeitsmarktsituation in den neuen EU-Mitgliedstaaten viele Frauen auf die Arbeitsuche nach Deutschland.

Infolge dieser Entwicklungen entstand in Deutschland ein grauer Arbeitsmarkt von Dienstleistungen im Bereich der häuslichen Betreuung und Pflege mit geschätzten 115.000 bis $300.000^{2}$ mittel- und osteuropäischen Arbeitskräften in deutschen Haushalten. Über die rechtliche Ausgestaltung dieser Beschäftigungsverhältnisse weiß man wenig, ebenso wie über die Vermittlungswege. Stattdessen berichten Angehörige in den Medien über ihre schwierige häusliche Pflegesituation, gelegentlich werden auch Lohndumping, prekäre Arbeit und Schwarzarbeit skandalisiert. Dem boomenden Markt der Vermittlungsagenturen schadet diese Berichterstattung wenig.

\section{Rechtliche Rahmenbedingungen der Arbeitszeit von Betreuungskräften im Haushalt}

Die Geltung des deutschen Arbeitszeitrechts im Beschäftigungsverhältnis einer im Haushalt ihres Arbeitgebers wohnenden Betreuungskraft ist zunächst nicht selbstverständlich. Denn $\$ 18$ Abs. 1 S. 3 ArbZG (Arbeitszeitgesetz) nimmt Arbeitnehmer, die in häuslicher Gemeinschaft mit den ihr anvertrauten Personen zusammenleben und sie eigenverantwortlich erziehen, pflegen oder betreuen, aus dem Anwendungsbereich des ArbZG aus.

Ob diese Ausnahme auch für die in Haushalten der Pflege- bzw. Betreuungsbedürftigen lebenden Arbeitskräfte gilt, ist bis heute höchstrichterlich nicht geklärt. Die Entstehungsgeschichte dieser Ausnahme sowie deren unverändert gebliebener Sinn und Zweck gebieten eine enge Auslegung: Im Zuge der Arbeiten am Arbeitszeitgesetz im Jahre 1994 wurde die ursprünglich geplante, auf der Vorgängerregelung der Arbeitszeitordnung (AZO) von 1938 gestützte Vorschrift, welche die Herausnahme aller im Haushalt Beschäftigten aus dem Anwendungsbereich des Arbeitszeitrechts vorsah, gestrichen. Zur Begründung hieß es in Beschlussempfehlung und Bericht des Ausschusses für Arbeit und Sozialordnung: „Die Mitglieder der Fraktionen der CDU/ CSU und FDP waren der Auffassung, daß das Gesetz auch auf im Haushalt beschäftigte Arbeitnehmer Anwendung finden solle, da den Beschäftigten im Haushalt der gleiche Schutz wie den übrigen Arbeitnehmern zuteil werden solle“.

Die dennoch eingeführte Ausnahme in der bis heute geltenden Fassung wurde wie folgt begründet: „Die besonderen Lebens- und Arbeitsbedingungen dieser Arbeitnehmer, z. B. Kinderdorfeltern in SOS-Kinderdörfern,

1 Der Beitrag baut auf den Erkenntnissen auf, die die Autorin im Rahmen ihrer Mitarbeit an einem von der Deutschen Forschungsgemeinschaft geförderten, von Prof. Dr. Christiane Brors an der Universität Oldenburg geleiteten Projekt zum Thema „Rechtliche Rahmenbedingungen des grenzüberschreitenden Personaleinsatzes aus Polen nach Deutschland am Beispiel der Pflegebranche" gewonnen hat.

2 Böning, M./Steffen, M. (2014): Migrantinnen aus Osteuropa in Privathaushalten: ver.di, Berlin.

3 Bundestagsdrucksache 12/6990, S. 40. 
lassen eine durch das öffentlich-rechtliche Arbeitszeitrecht zwingend vorgeschriebene Unterscheidung zwischen Freizeit und Arbeitszeit nicht zu. Damit wird zugleich einer Petition der SOS-Kinderdörfer Rechnung getragen“. ${ }^{4}$

Diese Rechtfertigung behält bis heute ihre Aktualität: Die Tätigkeit im SOS-Kinderdorf setzt eine Bereitschaft zum Aufbau einer elternähnlichen Beziehung gegenüber minderjährigen Schutzbefohlenen voraus, die eine zeitlich nicht einschränkbare Wahrnehmung von Fürsorge- und Schutzpflichten erfordert. Die Tätigkeit einer Betreuungskraft - ganz gleich ob in einer Einrichtung oder im Haushalt - kann hiermit nicht verglichen werden.

Zutreffend fordern Scheiwe und Schwach eine Differenzierung zwischen dem eigentlichen Anwendungsfall des $\$ 18$ Abs. 1 Nr. 3 ArbZG - den Kinderdorfeltern - und den Fällen von 24-Stunden-Pflege im Haushalt. ${ }^{5}$ Das Merkmal der Eigenverantwortlichkeit liegt nach dieser Auffassung bei den im Haushalt beschäftigten Betreuungskräften nicht vor, da sie - wie alle anderen Pflege- und Betreuungskräfte - bezüglich der Versorgung, Ernährung und Alltagshilfe der pflegebedürftigen Person angewiesen werden. ${ }^{6}$ Dagegen beruht das Konzept der SOS-Kinderdörfer auf dem selbstständig und frei gestalteten familienähnlichen Zusammenleben der „Eltern“ mit den Kindern und Jugendlichen, welches dem von Beziehungskontinuität geprägten Leben einer herkömmlichen Familie möglichst nahe kommen soll. ${ }^{7}$ In der häuslichen Pflege dagegen ist das Leben unter einem Dach nicht das Ziel, sondern ausschließlich die Nebenfolge der „Rund-um-die-Uhr“-Pflege, welche bereits durch die Einführung einer anderen Arbeitsorganisation beseitigt werden könnte. ${ }^{8}$ Für das Zusammenleben fehlt es hier zudem an der gemeinsamen Organisation des Lebensalltages und am gemeinsamen Wirtschaften. ${ }^{9}$ Auch darin unterscheiden sich die Kinderdorfhaushalte von den Altershaushalten, da in Ersteren die Betreuer nicht „bei“ den Kindern sondern „mit“ den Kindern zusammenleben. Zutreffend bezeichnet Scheiwe den $\$ 18$ Abs. 1 Nr. 3 ArbZG als „lex SOS-Kinderdorfmutter". ${ }^{10}$

Dieser engen Auslegung ist uneingeschränkt zuzustimmen. Unzutreffend ist dagegen die in der Literatur ohne jegliche argumentative Untermauerung vertretene Auffassung, welche zu den im Sinne des $\$ 18$ Abs. 1 Nr. 3 ArbZG „anvertrauten Personen“ neben den Kindern in SOS-Kinderdörfern auch Behinderte, Pflegebedürftige und ältere Menschen zählt. ${ }^{11}$

\section{Arbeitszeit der Betreuungskräfte im Haushalt als Politikum}

Im Angesicht der rasanten Entwicklung des Marktes der Rund-um-die-Uhr-Pflege erlangte die Frage der Anwendbarkeit von $\$ 18$ Abs. 1 Nr. 3 ArbZG auf die Fälle der Beschäftigung von sogenannten „live-in“-Betreuerinnen im Haushalt politische Brisanz. In einer kleinen Anfrage der Abgeordneten der Linksfraktion vom 14.12.2011 wollten diese wissen:

„Gilt auch bei Haushalts-/Pflegehilfskräften im Rahmen der vollen Arbeitnehmerfreizügigkeit das Arbeitszeitgesetz (ArbZG), und wenn ja, trifft es zu, dass nach $\$ 2$ AEntG grundsätzlich die Arbeitszeit von acht Stunden, in Ausnahmefällen von zehn Stunden Werktags, nach dem deutschen ArbZG bei Haushalts-/Pflegehilfskräften in privaten Haushalten einzuhalten ist, und wenn nein, welche Einschränkungen des $\$ 18$ ArbZG sind wirksam? ${ }^{\text {“12 }}$

Die Antwort der Bundesregierung (CDU-CSU/FDP) brachte zwar keine Klarheit, schloss jedoch die Anwendbarkeit der Ausnahmeregelung auf die Fälle der in Haushalten beschäftigten „live-in“-Pflege- und Betreuungskräfte nicht aus:

„[...] Sofern die Voraussetzungen (des $\$ 18$ Abs. 1 Nr. 3 ArbZG) vorliegen, findet das Arbeitszeitgesetz auch auf ausländische entsandte Arbeitnehmerinnen und Arbeitnehmer keine Anwendung. Bei der Beurteilung, ob eine Beschäftigte oder ein Beschäftigter nach $\$ 18$ Abs. 1 Nr. 3 ArbZG vom Anwendungsbereich des Arbeitszeitgesetzes ausgenommen werden kann, sind alle Umstände des Einzelfalls zu berücksichtigen. Daher ist eine generelle Aussage hierzu nicht möglich." ${ }^{\text {13 }}$

\section{Das Übereinkommen der ILO Nr. 189 und die Vorgehensweise der Bundesregierung}

Die Absicht, „live-in“-Betreuungs- und Pflegekräfte dem Schutzbereich des Arbeitszeitrechts zu entziehen, wird bei
4 Bundestagsdrucksache 12/6990, S. 44

5 Scheiwe, K./Schwach, V. (2013): Das Arbeitszeitrecht für Hausangestellte nach Ratifizierung der ILO-Konvention 189, in: Neue Zeitschrift für Arbeitsrecht 30 (20), S. $1116-1120$.

6 Scheiwe, K./Schwach, V. (2013), a. a. O., S. 1118; Heinlein, I. (2013): Kein Mindestlohn und unbegrenzte Arbeitszeit für häusliche Pflegerinnen?, in: Arbeit und Recht 61 (12), S. $469-475$.

7 Im Ergebnis ebenso: Heinlein, I. (2013), a. a. O., S. 474ff.; Buschmann/Ulber, ArbZG § 18 Abs. 4.
8 Scheiwe, K./Schwach, V. (2013), a. a. O., S. 1118

9 Anzinger/Koberski, ArbZG, § 18 Rn. 21; Buschmann/Ulber, ArbZG § 18 Rn. 4; ErfK/Wank, § 18 ArbZG Rn. 5.

10 Scheiwe, K./Schwach, V. (2013), a. a. O., S. $1118 f$.

11 ErfK/Wank, § 18 ArbZG Rn. 5.

12 Bundestagsdrucksache 17/8193, S. 3 Frage 6.

13 Bundestagsdrucksache $17 / 8373$, S. 4. 
aufmerksamer Lektüre des Entwurfs eines Gesetzes zum Übereinkommen Nr. 189 der Internationalen Arbeitsorganisation vom 16. Juni 2011 über menschenwürdige Arbeit für Hausangestellte ${ }^{14}$ deutlich. Deutschland hat das Übereinkommen im Jahre 2013 ratifiziert, im September 2014 trat dies in Kraft. Damit sind die darin enthaltenen Vorgaben für Deutschland rechtlich bindend.

Das Übereinkommen verpflichtet die Mitgliedstaaten, eine Gleichbehandlung von Haushaltsbeschäftigten mit anderen Beschäftigten u. a. in Bezug auf die normale Arbeitszeit und die täglichen und wöchentlichen Ruhezeiten sicherzustellen (Art. 10 Abs. 1). Dabei muss die wöchentliche Ruhezeit mindestens 24 aufeinanderfolgende Stunden betragen (Art. 10 Abs. 2). Zeiten, in denen Hausangestellte nicht frei über ihre Zeit verfügen können und sich zur Verfügung des Haushalts halten, um möglichen Aufforderungen Folge zu leisten, sind als Arbeitszeiten anzusehen (Art. 10 Abs. 3).

Art. 2 Abs. 2 Buchst. b) des Übereinkommens eröffnet den Mitgliedstaaten die Möglichkeit, „begrenzte Gruppen von Arbeitnehmern, bei denen besondere Probleme von erheblicher Bedeutung auftreten", aus dem Geltungsbereich des Übereinkommens auszuschließen. Hierzu führte die Bundesregierung aus:

„[...] Deutschland macht [...] von dieser Möglichkeit für die in $\$ 18$ Absatz 1 Nummer 3 des Arbeitszeitgesetzes (ArbZG) aufgeführte Personengruppe Gebrauch. [...] Eine Abgrenzung zwischen Freizeit und Arbeitszeit, wie in Artikel 10 Absatz 1 des Übereinkommens gefordert und wie sie das öffentlich-rechtliche Arbeitszeitrecht zwingend vorschreibt, ist hier nicht möglich “. ${ }^{15}$

Vergleicht man den Anwendungsbereich der Ausnahmevorschrift des $₫ 18$ Abs. 1 Nr. 3 ArbZG mit dem Geltungsbereich des Übereinkommens, wird klar, dass die Bundesregierung die „live-in“-Pflege- und Betreuungskräfte und nicht die SOS-Kinderdorf-Eltern meint. Denn das ILOÜbereinkommen gilt für Tätigkeiten, die „im Haushalt oder für den Haushalt einer anderen Person als der Hausarbeiterin durchgeführt werden “ ${ }^{16}$ In dem eigentlichen Anwendungsfall des $\$ 18$ Abs. 1 Nr. 3 ArbZG, nämlich bei der Tätigkeit der SOS-Kinderdorfeltern, handelt es sich nicht um einen Haushalt „einer anderen Person“. Für die SOS-Kinderdorfeltern gilt das Übereinkommen schlichtweg nicht.

Diese in der weiten Öffentlichkeit kaum wahrgenommene Diskussion bedeutet, dass politisch eine vollständige Entgrenzung der Arbeitszeit von ausländischen Betreuungsund Pflegekräften in deutschen Haushalten akzeptiert wird. Es wird hingenommen, dass für die in Haushalten arbeitenden und lebenden Pflege- und Betreuungskräfte weder die Grenze der höchstzulässigen Arbeitszeit noch die täglichen Ruhepausen und Ruhezeiten nach den Vorgaben des Arbeitszeitgesetzes gelten, sondern der Arbeitgeber diese selbstständig, im Rahmen der allgemeinen Schutzpflicht des $₫ 618$ Abs. 2 BGB bestimmen kann.

In Ermangelung gerichtlicher Klärung legt die Politik interessengeleitet das geltende Recht aus und überlässt den
Arbeitgebern die Entscheidung darüber, welche Arbeitszeiten mit der Sicherheit und Gesundheit der im Haushalt ihrer Arbeitgeber lebenden Beschäftigten vereinbar sind. Organisationen, die sich für Rechte von Hausangestellten einsetzen, gehen davon aus, dass die im Haushalt lebenden Betreuungskräfte aus dem Geltungsgereich des Übereinkommens ausgenommen wurden und appellieren an die Regierung, sie möge diese Ausnahme zurücknehmen. ${ }^{17}$

\section{Praxis der vertraglichen Arbeitszeitgestaltung}

Was von der Politik akzeptiert wird, setzt die Praxis eifrig um. Von der rechtlichen Unklarheit profitieren viele - die Vermittler, Betreuungsbedürftige und ihre Angehörigen nur nicht die Hausangestellten. Nachstehende Beispiele zeigen, wie die Entgrenzung der Arbeitszeit in der vertraglichen Praxis Einzug hält. Dabei muss eins beachtet werden: Vermittlung von Betreuungs- und Pflegekräften zur Direkteinstellung im Haushalt ist eine Seltenheit und wird von den Vermittlern als Qualitätsmerkmal hervorgehoben. Der Markt wird von sogenannten Pflegeagenturen aus Deutschland und dem Ausland beherrscht, die Dienstleistungsverträge mit den Haushalten schließen und sie mit dem Versprechen der „Rund-um-die-Uhr-Sorglosigkeit“ von den Lasten der Arbeitgeberrolle befreien. Dabei stehen die Betreuerinnen bei den Pflegeagenturen in der Regel als freie Mitarbeiterinnen unter Vertrag. Dass es sich hier um Umgehungstatbestände handelt, liegt auf der Hand: Die Weisungsstruktur und die Eingliederung sprechen für ein unmittelbares Arbeitsverhältnis zu der pflegebedürftigen Person oder ihren Angehörigen. Aber auch hier gilt: Wo kein Kläger, da kein Richter.

$\mathrm{Zu}$ den wenigen Anbietern, die Pflege- und Betreuungskräfte zur Direkteinstellung an Haushalte vermittelten, gehörte die Caritas. Mit seinem Projekt Caritas24 wollte der Verband eine Alternative zu den Beschäftigungsverhältnissen in einer rechtlichen Grauzone oder in

14 Bundestagsdrucksache 17/12951.

15 Bundestagsdrucksache 17/12951, S. 18; wortlautidentisch: Bundesratdrucksache 114/13 S. 18.

16 Art. 1 Buchst. a) des Übereinkommens, Bundestagsdrucksache 17/12951; Kocher, E. (2012): Hausarbeit als Erwerbsarbeit: Der Rechtsrahmen in Deutschland: Voraussetzungen einer Ratifikation der ILO-Domestic Workers Convention durch die Bundesrepublik Deutschland, Gutachten im Auftrag der Hans-Böckler-Stiftung, http://www.boeckler.de/ pdf_fof/S-2012-524-3-1.pdf.

17 Deutscher Frauenrat (2013): Beschlüsse der Mitgliederversammlung 2013, http://www.frauenrat.de/fileadmin/user_ upload/frauenrat/beschluesse/Beschluesse_2013-web.pdf. Zentralkomitee der deutschen Katholiken (2013): Stellungnahme "Schutz für Hausangestellte", http://www.zdk.de/ve roeffentlichungen/salzkoerner/detail/Schutzrechte-fuerHausangestellte-613V. 
der Illegalität anbieten und gleichermaßen die Haushalte und Pflegerinnen unterstützen. Für die vermittelnden Arbeitsverträge zwischen der Betreuungskraft und dem Pflegebedürftigen verwendete Caritas 24 Vertragsformulare der Zentralen Auslands- und Fachkräftevermittlung (ZAV) der Bundesagentur für Arbeit, jedoch mit einer Änderung, welche nicht als unwesentlich bezeichnet werden kann. Wortlautidentisch wurde im deutsch-polnischen Vertragsformular die wöchentliche Arbeitszeit auf 38,5 Stunden festgelegt. Anschließend enthielt der Vertrag folgende Klausel: Die Aufnahme in die häusliche Gemeinschaft schließt die Anwendung des Arbeitszeitgesetzes (ArbZG) aus ( $\$ 18$ Abs.1 Nr. 3 ArbZG). Die Klausel wurde ins Polnische folgend übersetzt: „Wstąpienie do wspólnoty domowej wyklucza zastosowanie niemieckiego prawa pracy“ ( $\$ 18$ ust. $1 \mathrm{nr} 3$ niemieckie prawo pracy).

Auf Deutsch bedeutet dies wiederum: „Die Aufnahme in die häusliche Gemeinschaft schließt die Anwendung des deutschen Arbeitsrechts aus ( $\$ 18$ Abs. 1 Nr. 3 des deutschen Arbeitsrechts)“. Ob es sich an dieser Stelle um einen Flüchtigkeitsfehler der Übersetzung oder eine bewusste Sinnverstellung handelt, ist von zweitrangiger Bedeutung. Sicher ist, dass während der Laufzeit des Projektes von 2011 bis einschließend Februar 2014 die von der Caritas verwendeten Musterverträge diese Klausel enthalten haben. Diese Passage ist außerdem die Einzige, die sich - sowohl hinsichtlich des Wortlauts als auch im Hinblick auf die Struktur - von dem zweisprachigen ZAV-Vordruck unterscheidet.

Dass es sich nicht um einen Paragraphen des deutschen Arbeitsrechts handeln kann, sondern höchstens um einen eines konkreten Gesetzes, kann eine polnische Betreuerin in aller Regel nicht erkennen. Ebenso wenig ist zu erwarten, dass der deutschsprachige Anwender, also die zu betreuende Person oder ihre Angehörigen als Arbeitgeber erkennen, dass die Geltung der Ausnahmevorschrift in dem von ihnen geschlossenen Arbeitsverhältnis höchst problematisch ist. Entscheidend ist, dass diese Formel bei juristischen Laien auf beiden Seiten des Arbeitsverhältnisses den Eindruck erwecken muss, das deutsche Recht, zumindest aber das deutsche Arbeitszeitrecht, findet zwischen ihnen keine Anwendung. Aus Sicht der Arbeitnehmerin wird dadurch der Sinn des gesamten Vertrages, nämlich eine Zusicherung von Arbeitsbedingungen im Einklang mit den Schutzvorschriften des deutschen Arbeitsrechts, verstellt. Es ist lebensfremd zu glauben, dass eine Beschäftigte, nachdem sie die vermeintliche Nichtanwendbarkeit des deutschen Arbeitsrechts zur Kenntnis genommen und den Vertrag in dieser Form unterschrieben hat, anschließend gegen ihren Arbeitgeber eventuelle Ansprüche geltend machen wird. Der deutsche Arbeitgeber bekommt mitgeteilt, dass er sich bei der Inanspruchnahme der Leistungen der Hausangestellten an keine zeitlichen Schranken halten muss.

Ebenso wie Caritas24 verfolgt die Stiftung Europäische Begegnung (SEB), Betreiber der Homepage www.curae.de, primär ideelle Ziele - es gehe „um Völkerverständigung“ - und preist das eigene Angebot als "gesetzestreu“ an. Auch
SEB vermittelt Betreuungskräfte zur Direkteinstellung im Haushalt und stellt hierzu deutsch-polnische Arbeitsvertragsformulare zur Verfügung. Diese enthalten folgende Regelung zur Arbeitszeit: (Originalschreibweise):

„Die Arbeitszeit ist gem. Arbeitszeitschutzgesetz $\$ 18$ Abs. 1 Ziffer 3 für Arbeitnehmer in der häuslichen Gemeinschaft nicht geregelt. Sie wird durch den AG entsprechend den Bedürfnissen des $\mathrm{HH}$ im Zeitraum morgens $7 \mathrm{Uhr}$ bis abends 20 Uhr festgelegt." Von arbeitsfreien Tagen ist weder im Vertrag noch - nach Schilderungen der von SEB vermittelten Pflegekräfte - in der Praxis die Rede. Schöpft der Arbeitgeber den Rahmen des Arbeitsvertrages aus, kann eine Beschäftigung im Umfang von 91 Stunden/Woche die Folge sein.

Caritas24 und SEB sind mit ihrem Angebot zur Direkteinstellung eine Ausnahme auf dem Markt der Pflegedienstleistungen. In der großen Mehrheit der Fälle werden dem Einsatz von Betreuungs- und Pflegekräften freie Dienstleistungsverträge zugrunde gelegt - sowohl im Verhältnis zwischen den Vermittlungsagenturen und den deutschen Kunden als auch zwischen den Agenturen und den Betreuungs- bzw. Pflegekräften. Hier erfolgt die Entgrenzung der Arbeitszeit nicht durch den Hinweis auf die angebliche Nichtanwendbarkeit des Arbeitszeitgesetzes, sondern nimmt direkte Formen an. Klauseln wie: „Dienstzeit besteht aus 24-Stunden-Rufbereitschaft zur Hilfe und Betreuung der Person des Leistungsnehmers in seinem eigenen Zuhause“ oder Vertragsstrafen für das „Entfernen vom Leistungsort ohne Erlaubnis des Leistungsempfängers" sind keine Seltenheit und zeigen das Ausmaß der einseitigen Herrschaftsausübung in diesen formell freien, zivilrechtlichen Vertragsverhältnissen.

\section{Ausblick}

Deutsche Haushalte sind für die dort beschäftigten ausländischen Betreuungs- und Pflegekräfte ein schutzloser Raum. Im Zusammenhang mit der Ratifizierung des Hausangestellten-Übereinkommens Nr. 189 war Deutschland verpflichtet, die Rechtslage und die faktische Situation in seinem Staatsgebiet in Einklang mit dem Übereinkommen zu bringen. Dies ist bislang nicht geschehen. Mit ihrer Haltung in der Frage der Arbeitszeit stellt die Politik ausgerechnet diejenigen schutzlos, die des Schutzes am stärksten bedürfen. Die Praxis zieht daraus ihre Schlüsse. Es ist längst an der Zeit, diesen Zustand zu beenden.

\section{AUTORIN}

MARTA BÖNING, Dr., ist Referatsleiterin in der Abteilung Recht beim Bundesvorstand des Deutschen Gewerkschaftsbundes (DGB). Arbeitsschwerpunkt: Fragen des Individualarbeitsrechts.

marta.boening@dgb.de 